\title{
Optimization of culture conditions to obtain maximal growth of penicillin-resistant Streptococcus pneumoniae
}

\author{
Andrea V Restrepo ${ }^{1,2}$, Beatriz E Salazar ${ }^{1}$, María Agudelo ${ }^{1}$, \\ Carlos A Rodriguez ${ }^{1,3}$, Andres F Zuluaga ${ }^{1,3}$ and Omar Vesga*1,2
}

\begin{abstract}
Address: ${ }^{1}$ GRIPE: Grupo Investigador de Problemas en Enfermedades Infecciosas, University of Antioquia Medical School, Medellín, Colombia, ${ }^{2}$ Section of Infectious Diseases, Department of Internal Medicine, University of Antioquia Medical School, Medellín, Colombia and ${ }^{3}$ Department of Pharmacology \& Toxicology, University of Antioquia Medical School, Medellín, Colombia

Email: Andrea V Restrepo - cavanegas@epm.net.co; Beatriz E Salazar - salazarbe@yahoo.com; María Agudelo - mariaag10@yahoo.com; Carlos A Rodriguez - andreios@yahoo.com; Andres F Zuluaga - azuluaga@acin.org; Omar Vesga* - ovesga@epm.net.co

* Corresponding author

Published: 02 June 2005

BMC Microbiology 2005, 5:34 doi:10.1/86/147|-2180-5-34

This article is available from: http://www.biomedcentral.com/I47I-2/80/5/34

(c) 2005 Restrepo et al; licensee BioMed Central Ltd.

This is an Open Access article distributed under the terms of the Creative Commons Attribution License (http://creativecommons.org/licenses/by/2.0), which permits unrestricted use, distribution, and reproduction in any medium, provided the original work is properly cited.

Received: 25 February 2005

Accepted: 02 June 2005
\end{abstract}

\begin{abstract}
Background: Streptococcus pneumoniae, particularly penicillin-resistant strains (PRSP), constitute one of the most important causes of serious infections worldwide. It is a fastidious microorganism with exquisite nutritional and environmental requirements to grow, a characteristic that prevents the development of useful animal models to study the biology of the microorganism. This study was designed to determine optimal conditions for culture and growth of PRSP.

Results: We developed a simple and reproducible method for culture of diverse strains of PRSP representing several invasive serotypes of clinical and epidemiological importance in Colombia. Application of this 3-step culture protocol consistently produced more than $9 \log _{10} \mathrm{CFU} / \mathrm{ml}$ of viable cells in the middle part of the logarithmic phase of their growth curve.

Conclusion: A controlled inoculum size grown in 3 successive steps in supplemented agar and broth under $5 \% \mathrm{CO}_{2}$ atmosphere, with $\mathrm{pH}$ adjustment and specific incubation times, allowed production of great numbers of PRSP without untimely activation of autolysis mechanisms.
\end{abstract}

\section{Background}

Streptococcus pneumoniae, one of the most important human pathogens of all times, is today an even more serious menace because of its increasing resistance to penicillin, the first-choice antibiotic [1]. It is a fastidious organism to grow both in vitro and in animal infection models. Probably because of the wide genetic diversity of this organism and its exclusive nature as human pathogen, it is difficult to obtain comparable cell production when culturing different strains even within the same serotype [2].
In the field of experimental animal models there is also wide variation in the susceptibility of mice strains and specific organs to infection with $S$. pneumoniae $[3,4]$. While new drugs with activity against penicillin-resistant S. pneumoniae (PRSP) are being discovered, only penicillin-susceptible strains grow in the lungs of experimental animals with some consistency [5]. Although other organs 
such as the thighs of neutropenic mice are susceptible to

Table I: Serotypes and minimal inhibitory and bactericidal concentrations (MIC / MBC) of six antimicrobial agents against nine strains of Streptococcus pneumoniae

\begin{tabular}{|c|c|c|c|c|c|c|c|}
\hline \multirow[t]{2}{*}{ Strains } & \multirow[t]{2}{*}{ Serotype } & \multicolumn{3}{|c|}{ MIC / MBC $[\mu \mathrm{g} / \mathrm{mL}]$} & \multicolumn{3}{|c|}{ MIC* $[\mu \mathrm{g} / \mathrm{mL}]$} \\
\hline & & PEN & CRO & VAN & ERY & CHL & SXT \\
\hline INS-E6II I & $6 \mathrm{~B}$ & $2.00 / 2.00$ & $1.00 / 1.41$ & $0.35 / 0.35$ & 0.03 & 32.00 & 4.00 \\
\hline INS-E674 & 14 & $2.00 / 2.00$ & $1.00 / 1.00$ & $0.25 / 0.25$ & 0.06 & 4.00 & 8.00 \\
\hline INS-E676 & 14 & $1.00 / 1.41$ & $1.00 / 1.41$ & $0.35 / 0.70$ & 0.06 & 4.00 & 8.00 \\
\hline INS-E678 & 14 & $0.12 / 0.12$ & $0.03 / 0.03$ & $0.25 / 0.25$ & 0.06 & 4.00 & 16.00 \\
\hline INS-E682† & $6 \mathrm{~B}$ & $0.01 / 0.01$ & $0.01 / 0.01$ & $0.25 / 0.25$ & 0.06 & 32.00 & 0.50 \\
\hline INS-E683 & $9 \mathrm{~V}$ & $1.00 / 1.00$ & $0.35 / 0.35$ & $0.35 / 0.35$ & 0.06 & 4.00 & 32.00 \\
\hline INS-E684 & 14 & $1.00 / 1.00$ & $0.50 / 0.50$ & $0.25 / 0.25$ & 0.13 & 4.00 & 1.00 \\
\hline INS-E685† & 1 & $0.01 / 0.01$ & $0.01 / 0.01$ & $0.25 / 0.25$ & 0.06 & 2.00 & 0.50 \\
\hline ATCC 49619 & $19 \mathrm{~F}$ & $0.46 / 0.46$ & $0.06 / 0.12$ & $0.33 / 0.44$ & 0.06 & 4.00 & 0.25 \\
\hline
\end{tabular}

$\mathrm{PEN}=$ penicillin; $\mathrm{CRO}=$ ceftriaxone; $\mathrm{VAN}=$ vancomycin; $\mathrm{CHL}=$ chloramphenicol; $\mathrm{ERY}=$ erythromycin; $\mathrm{SXT}=$ trimethoprim-sulfamethoxazole (only first component values shown)

* MIC determined by Instituto Nacional de Salud (INS), Bogotá, Colombia.

† Breakpoint for penicillin-susceptible strains: MIC $\leq 0.06 \mu \mathrm{g} / \mathrm{mL}$.

progressive infection with PRSP, a reproducible pneumonia model remains elusive for most strains of PRSP.

Little attention has been given to the culture conditions of $S$. pneumoniae, critical to obtain the appropriate amount of viable log-phased cells to inoculate the animals. $S$. pneumoniae was reclassified within the group of anaerobic bacteria because its relatively small genome simply lacks many genes required for aerobic growth [6]. As a Grampositive catalase-negative coccus that generates $\mathrm{H}_{2} \mathrm{O}_{2}$ via a flavoenzime system, it grows better in presence of a source of catalase such as red blood cells $[7,8]$. In contrast to most bacteria, $S$. pneumoniae also requires choline for growth in defined media, and reducing agents are almost essential. Growth of most strains requires vitamin B complex (biotin, nicotinamide, pantothenate, pyridoxal, riboflavin and thiamine), adenine, guanine, uracil, and 7-10 amino acids. Its energy metabolism is fermentative, yielding primarily low levels of lactic acid, but optimum $\mathrm{pH}$ for growth is 7.8 with a range of $6.5-8.3$ [9]. Besides these limitations, $S$. pneumoniae displays a particularly effective quorum sensing system that activates several potent autolysins once certain conditions are met within the growing population, and this is perhaps the most important hurdle to overcome when trying to obtain viable logphased cells [10]. Among several factors not well understood, culture acidification is one of the conditions met by the growing population that clearly contributes to start the autolysis process [11].

We hypothesized that optimization of culture conditions tending to prevent environmental acidification and its consequent activation of autolysis mechanisms could lead to better production of PRSP in vitro, and aimed to determine and provide such ideal conditions for the growth of this microorganism, so they could be applied to diverse strains of clinical importance to develop a reproducible animal model of pneumonia.

\section{Results}

\section{Susceptibility testing}

Minimal inhibitory (MIC) and bactericidal (MBC) concentrations of six antimicrobial agents for these strains are shown in Table 1. Reduced susceptibility to penicillin was demonstrated for 6 strains, the other 2 were susceptible. Among those non-susceptible, 4 strains displayed intermediate (MIC $0.12-1 \mu \mathrm{g} / \mathrm{mL}$ ) and 2 full resistance to penicillin (MIC > $1 \mu \mathrm{g} / \mathrm{mL}$ ). Only one strain (INS-E685) was susceptible to all six antibiotics tested, but all of them were susceptible to erythromycin and vancomycin. MIC of ceftriaxone against PRSP INS-E611, E674 and E676 was $1 \mu \mathrm{g} / \mathrm{mL}$, a value conferring intermediate resistance for CSF isolates, as was the case for strain INS-E676 (Table 1).

\section{Evaluation of baseline culture variables}

The results are summarized in Table 2. For cryoprotection, skim milk invariably allowed recuperation of frozen organisms, while $17 \%$ glycerol failed once (no viable bacteria after thawing). During Phase 0, we found that two successive passes on solid media were necessary for complete recuperation after thawing, and that their optimal incubation time was 15 hours. After 15 hours colony umbilication became deeper, suggesting progression of the autolysis process [9]. The number of colonies from 
Table 2: Comparison of diverse culture variables for productivity of PRSP cells

\begin{tabular}{llll}
\hline \multicolumn{1}{c}{ Variable } & \multicolumn{1}{c}{ Phase 0 } & Phase I & Phase 2 \\
\hline Cryoprotection Media & SM $>$ I7\%G:TSB & NA & NA \\
Inoculum (colonies) & NA & NA $>5$ or I5 or loopful & THB $>$ BHI \\
Culture Broth & NA & THB $>$ BHI & $2.0>0$ \\
Yeast Extract (\% YE) & NA & $2.0>0$ & $2.5>0$ \\
Horse Blood (\% HB) & NA & $2.5>0$ & qlh $>$ NoAdj \\
PH adjustment to 7.8 & I5 $>18>24$ & Initially $>$ NoAdj & $3 *, 5 \dagger, 6 \neq$ \\
Incubation time (h) & NA & $12>15,18,24$ & $6=7=8$ \\
Ideal dilution (Tube \#) & & $5>4>1-3$ & \\
\hline
\end{tabular}

SM = Skim Milk; > implies "superior to"; NA = non applicable to that Phase; 17\%G:TSB = 17\% glycerol in trypticase soy broth; THB = Todd Hewitt Broth; $\mathrm{BHI}=$ Brain Heart Infusion; NoAdj= no adjustment; *Tube 6; †Tube 7; $\ddagger$ Tube 8.

Table 3: Influence of inoculum size in cell production of Streptococcus pneumoniae INS-E6 I I after I 2 hours of Phase I culture in supplemented THB

\begin{tabular}{cccr}
\hline Number of CFU (Phase 0) & $\mathrm{OD}_{600 \mathrm{~nm}}($ Phase I) & Broth pH (Phase I) & Phase I Production [log $\left.{ }_{10} \mathrm{CFU} / \mathrm{mL}\right]$ \\
\hline 1 & 0.381 & 7.90 & 7.278 \\
5 & 1.878 & 7.00 & 8.662 \\
10 & $0.914-1.884$ & $7.08-7.32$ & $8.884 \pm 0.047$ \\
15 & 1.766 & 6.28 & 9.067 \\
Loopful & 1.778 & 6.17 & 8.851 \\
\hline
\end{tabular}

Phase 0 employed to inoculate Tube 1 in Phase 1 was critical to maximize the number of viable log-phased cells: 10 colonies into $10 \mathrm{ml}$ of culture broth gave the best results. Since broth acidification is known to start autolysis, a low pH after 12 hours of incubation during Phase 1 was used as an indicator of poor viability. Compared with 10 colonies, 5 or less produced lower cell numbers with similar decrease in $\mathrm{pH}$, and 15 or more colonies gave greater cell numbers but with a profound decrease in $\mathrm{pH}$ (Table 3 ). Besides 5\% sheep blood, additional supplementation of trypticase soy agar (TSA) with $0.5 \%$ yeast extract (instead of $0 \%$ ) during Phase 0 increased the number of viable cells produced by broth culture in Phase 1 from $6.60-7.60$ to $8.32-8.63 \mathrm{CFU} / \mathrm{ml}$.

The type of liquid media was tested before (standard broth) and after diverse adjustments (supplemented broth). Standard Todd Hewitt Broth (THB) was more reliable than Brain Heart Infusion (BHI) made by Oxoid and BBL, because it consistently produced 1.63-2.64 $\log _{10}$ $\mathrm{CFU} / \mathrm{ml}$ per hour with all strains and lots tested. BBL-BHI production was low (1.34-1.63 $\log _{10} \mathrm{CFU} / \mathrm{ml}$ per hour) from the beginning and not used further. BHI Oxoid was similar to THB in cell production, but not reproducible with the different lots tested to grow strain INS-E611 (Figure 1). Analysis of these four media by repeated measures ANOVA demonstrated a non-significant difference $(\mathrm{P}=$
0.7976), as expected from the similarity in production during the first few hours of cultivation. However, as illustrated by Figure 2, productivity at the end of each culture (5 hours) was significantly better with supplemented THB compared with standard THB and BHI $(\mathrm{P}=0.0022$, one way ANOVA). While stationary and death phase with $\leq$ $8.0 \log _{10} \mathrm{CFU} / \mathrm{ml}$ was reached before 3 hours with standard broths, supplementation of THB consistently produced $\geq 9.0 \log _{10} \mathrm{CFU} / \mathrm{ml}$ and prolonged logarithmic growth during Phase 2 up to 4 hours in Tube 6, 5 hours in Tube 7 , and 7 hours in Tube 8 (Figure 3). Incubation time during Phase 1 was best at 12 hours, with longer periods resulting in poor growth during Phase 2, mainly determined by media acidification. Adjustment of $\mathrm{pH}$ was fundamental during both Phase 1 and Phase 2. For practical reasons, Phase 1 broth was adjusted at the beginning of the 12-hour culture, but Phase 2 broth was adjusted every hour. In Figures 1, 2, 3, the term "supplemented" also implies this protocol for $\mathrm{pH}$ adjustment.

\section{Supplementation and optimization of culture conditions for PRSP}

Optimized culture conditions produced $\geq 9 \log _{10} \mathrm{CFU} / \mathrm{ml}$ of log-phased cells in Phase 2. These results were reproducible with all PRSP strains tested (Figure 4) under the following protocol: 


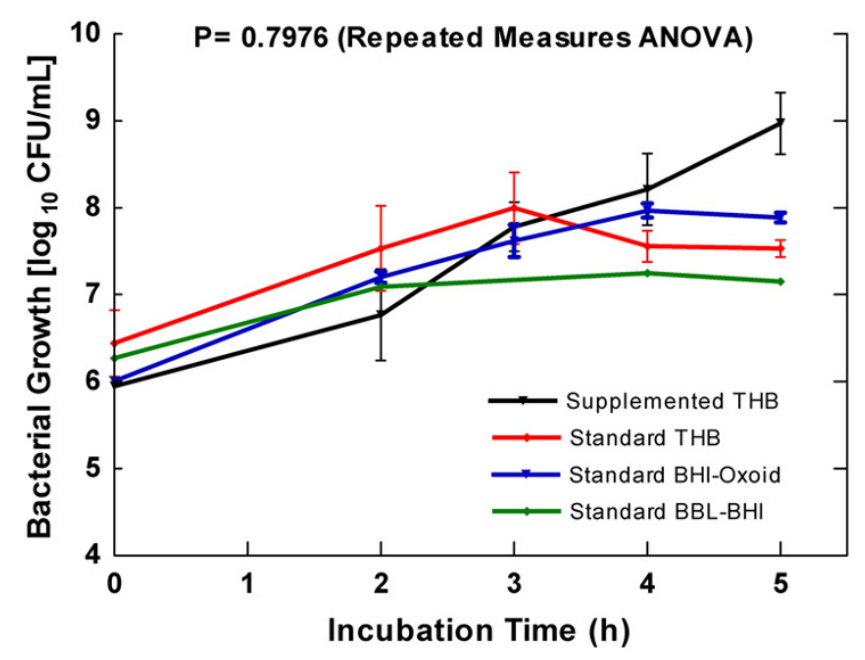

Figure I

Comparison of three standard liquid media with a supplemented medium for culture of $S$. pneumoniae INS-E6 I I. Five-hour growth curves for a PRSP strain in four different culture media: standard BHI (two brands), standard THB, and supplemented THB. All curves were obtained from Tube 7 (Phase 2). Bacterial growth was poor in standard $\mathrm{BBL}-\mathrm{BHI}$, and was not used for further experiments. As expected, there was no difference among these media when every data point along time was compared by repeated measures ANOVA ( $P=0.7976)$. However, there was a significant difference in maximal growth between supplemented THB and standard media, as illustrated by Figure 2 .

Phase 0: from frozen stock (skim milk), cells are resuscitated by two consecutive passes on 5\% sheep blood TSA supplemented with $0.5 \%$ yeast extract and incubated for 15 hours under $5 \% \mathrm{CO}_{2}$ atmosphere.

Phase 1: 10 colonies from the second plate are inoculated into $10 \mathrm{ml}$ of supplemented THB (Tube 1), followed by 4 successive 1:10 dilutions into identical liquid media (Tubes 2-5). Supplementation implies pH adjustment to 7.8 with $1 \mathrm{~N} \mathrm{NaOH}$ after addition of $2 \%$ yeast extract and $2.5 \%$ horse blood. All 5 tubes are incubated for 12 hours under $5 \% \quad \mathrm{CO}_{2}$ atmosphere, without additional $\mathrm{pH}$ adjustment.

Phase 2: $1 \mathrm{ml}$ of bacterial suspension taken from the most diluted tube with visible growth from Phase 1 is inoculated into $9 \mathrm{ml}$ of supplemented THB (Tube 6) in Phase 2. With most strains and under conditions just described for Phases 0 and 1, Tube 5 or 4 should have visible growth by the end of Phase 1 . Tubes 3, 2, and 1 should not be used, because their $\mathrm{pH}$ is invariably acidic by the end of Phase 1 , usually giving very low cell counts during Phase 2 . After inoculation of Tube 6 , two successive 1:10 dilutions are made into identical media (Tubes 7 and 8). Each one of these 3 tubes is incubated under $5 \% \mathrm{CO}_{2}$ atmosphere, and their $\mathrm{pH}$ is adjusted every hour to 7.8 with $1 \mathrm{~N} \mathrm{NaOH}$. We found that optimal incubation periods were 4, 5 and 7 hours for Tubes 6, 7, and 8, respectively.

\section{Discussion}

Our results demonstrate that optimization of culture conditions is an easy, non-expensive and reproducible way to attain penicillin-resistant $S$. pneumoniae growth over 9.5 $\log _{10} \mathrm{CFU} / \mathrm{ml}$ without the need of more complex methods (e.g. chemostat).

The S. pneumoniae serotypes used in this study are representative of the most frequent invasive isolates from blood and CSF in Colombia [20,21].

The three standard media used initially (BHI-Ox, BBL-BHI and THB) are recommended by American Society for Microbiology and Clinical Laboratory Standards Institute (CLSI, formerly NCCLS) for S. pneumoniae growth, but their productivity was quite different. In contrast with $\mathrm{BHI}, \mathrm{THB}$ has soy bean peptone and higher $\mathrm{pH}$ (7.4 vs 7.8, respectively). Conversely, BBL-BHI has the highest dextrose concentration $(3 \mathrm{~g} / \mathrm{L})$, which results in marked acidification of the media as anaerobic growth progresses [2]. In this aspect, we confirmed previous findings of acidic $\mathrm{pH}$ (under 6.5) inhibiting growth of penicillin resistant and susceptible $S$. pneumoniae strains [12]. To avoid media acidification, we limited the number of colonies produced during Phase 0 to inoculate supplemented THB in Tube 1 (Phase 1) and adjusted $\mathrm{pH}$ to 7.8 at the beginning of Phase 1 and every hour during Phase 2. Since $\mathrm{pH}$ control is not enough to attain growth over $8.7 \log _{10} \mathrm{CFU} / \mathrm{ml}$ [12], we addressed two additional characteristics that influence S. pneumoniae growth: autolysis and competence. The process of autolysis takes place around the upper part of the logarithmic phase of growth, is started by pheromones liberated by a complex quorum sensor system, and is characterized by extensive cellular death $[10,11]$. Competence, or the capacity for genetic transformation, is well developed in S. pneumoniae, and particularly well in PRSP, but it does not happen without repercussion in bacterial growth, both in vitro and in vivo $[3,5,13]$. Although the relationship between autolysis and competence not always goes in the same direction, choline mutants (the target of most autolysins) have shown important changes respect to wild type pneumococci, including abnormal growth in long chains instead of diplococci, loss of competence for transformation, resistance to autolysis, and resistance to cell-wall active antibiotics [14]. Based on these facts, we hypothesized that prevention of autolysis could improve production of viable cells even in the logarithmic phase of bacterial growth. Besides offering a suitable culture media, shorter incubation times and separate 


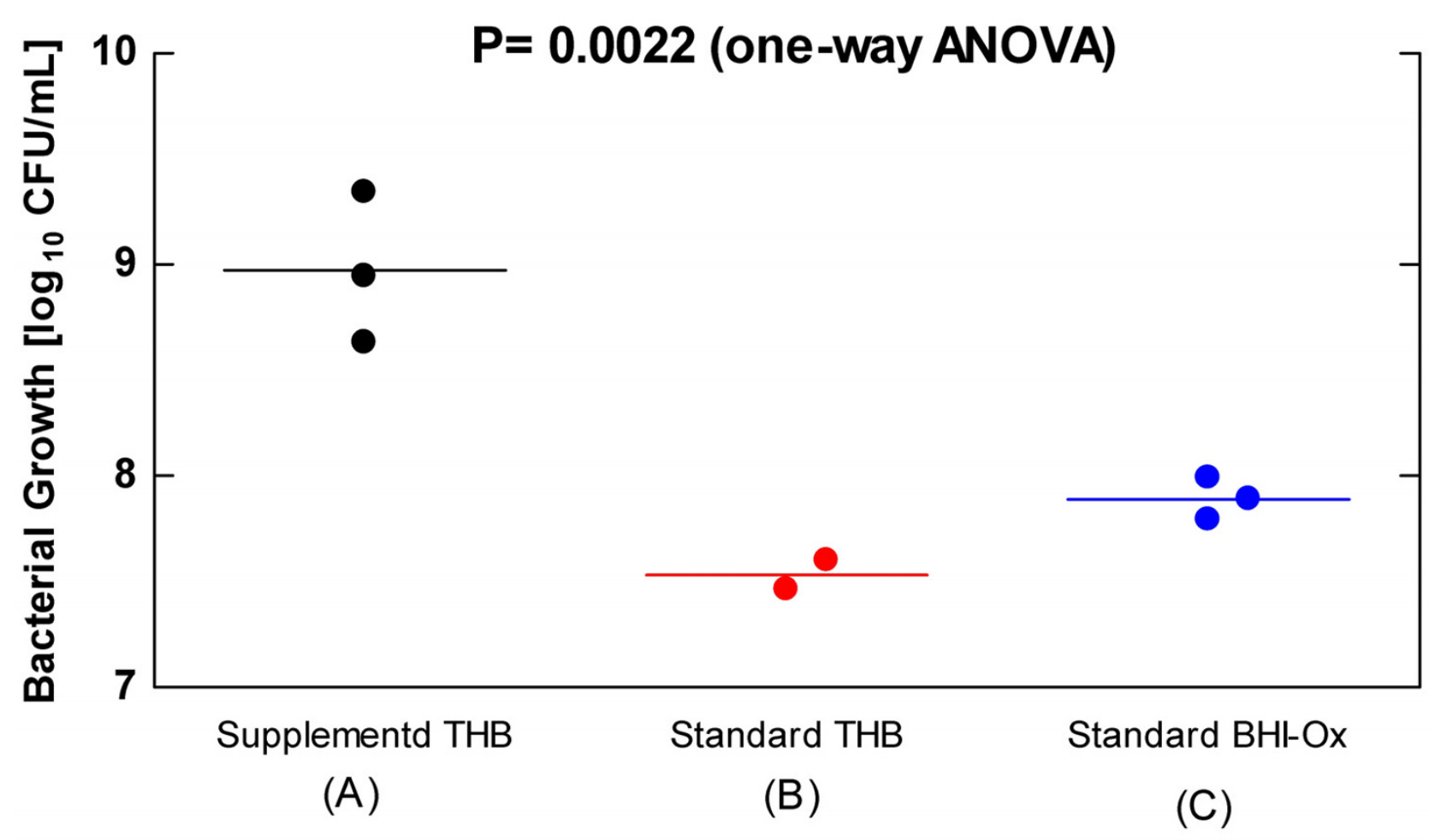

\begin{tabular}{l|c|c|c}
\hline \multirow{2}{*}{ Group } & $\begin{array}{c}\text { Bonferroni's Multiple Comparison Test } \\
\text { Mean difference in } \\
\text { growth [ } \log _{10} \text { CFU/mL] }\end{array}$ & $\begin{array}{c}\mathbf{9 5 \%} \text { Cl of difference } \\
{\left[\log _{10} \text { CFU/mL] }\right.}\end{array}$ & P value \\
\hline A vs B & 1.44 & 0.6723 to 2.210 & $P<0.01$ \\
A vs C & 1.08 & 0.3945 to 1.770 & $P<0.01$ \\
B vs C & -0.36 & -1.128 to 0.4100 & $P>0.05$ \\
\hline
\end{tabular}

Figure 2

Maximal growth of S. pneumoniae INS-E6 I I cultured in three liquid media. The graph illustrates cell production of each medium after 5 hours of incubation; all cultures were obtained from Tube 7 (Phase 2). Supplemented THB was clearly superior to standard $\mathrm{THB}$ and $\mathrm{BHI}$.

culture process in three phases allowed generous bacterial growth before triggering autolysis mechanisms. Given that concentrations of $8 \log _{10} \mathrm{CFU} / \mathrm{ml}$ were obtained $1-2$ hours before reaching the maximum incubation periods shown in Figure 3, inoculation of healthy cells in large numbers is possible before triggering autolysis and, probably, while pneumococci are still virulent enough to induce acute pneumonia in animal models.

Goncalves et al specifically addressing the optimal growing conditions for $S$. pneumoniae, described a batch cultivation procedure for serotype $23 \mathrm{~F}$, suitable for large scale polysaccharide production to manufacture vaccines [2].
They used Hoeprich's medium with different glucose concentrations and supplemented with various amino acids and a vitamin solution, and provided an anaerobic environment with $\mathrm{N}_{2}$ or $\mathrm{CO}_{2}$. Their results, expressed in terms of biomass and polysaccharide production, are difficult to compare with ours. Moreover, they used autolysis as a way of releasing polysaccharide, whereas we tried to avoid this process to obtain viable bacteria.

This is the first report on culture optimization for maximum growth of PRSP with simple methods, and is directed to facilitate the use of these strains in experimental procedures such as the development of animal models 


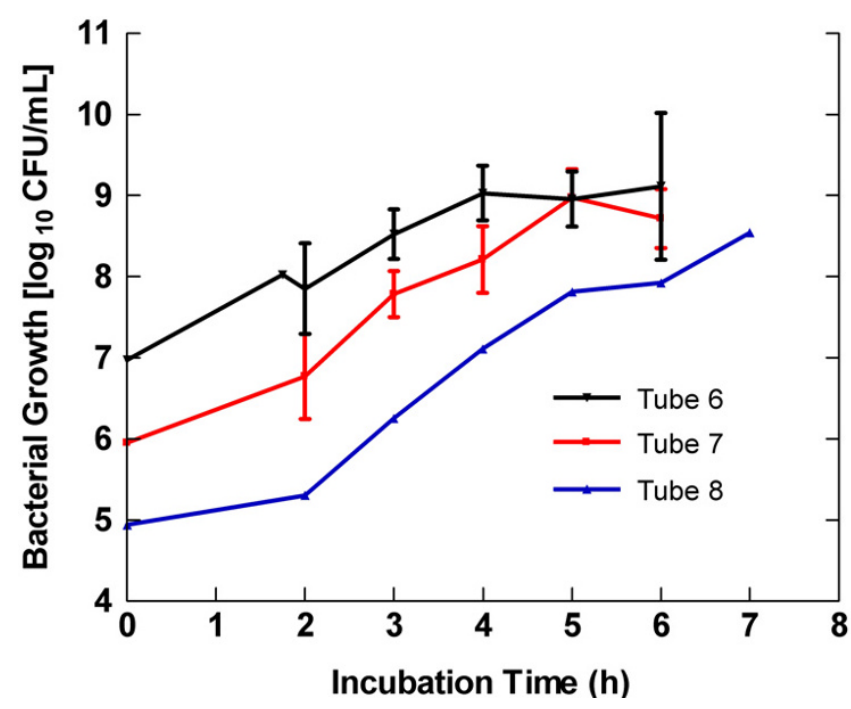

Figure 3

Growth of S. pneumoniae INS-E6 I I during Phase 2 in supplemented THB. One-mL bacterial suspension produced by Tube 5 (Phase I) after $12 \mathrm{~h}$ of incubation was inoculated into 9-mL fresh broth (Tube 6, I:10 dilution) and diluted further into Tube 7 ( $1: 100)$ and Tube 8 ( $1: 1000)$. Incubation under $5 \% \mathrm{CO}_{2}$ atmosphere and $\mathrm{pH}$ adjustment every hour followed for 5-6 hours, with simultaneous plating for CFU counting. The graph illustrates the time to reach maximum growth for each inoculum size before triggering autolysis: 4, 5 and 7 hours for Tubes 6, 7 and 8, respectively. With this method, very similar maximum growths were attained under different inoculum sizes. Experiments with each tube were done $\mathrm{I}-3$ times.

of pneumonia. The cultivation conditions described here generate high concentrations of log-phased bacteria without early and inconvenient induction of autolysis.

\section{Conclusion}

In this study we demonstrate that a standardized inoculum grown in supplemented solid and liquid media with $\mathrm{pH}$ adjustment and control of incubation times in three phases produces viable PRSP far beyond the limiting 8 $\log _{10} \mathrm{CFU} / \mathrm{mL}$. This method should allow improvement in experimental approaches to solve important questions regarding the biology, pathology, and therapeutics of PRSP.

\section{Methods}

\section{Bacterial strains}

Eight clinical strains of Streptococcus pneumoniae, obtained from very sick patients nationwide, were supplied by the Colombian Instituto Nacional de Salud (INS). These included six strains non susceptible to penicillin (called

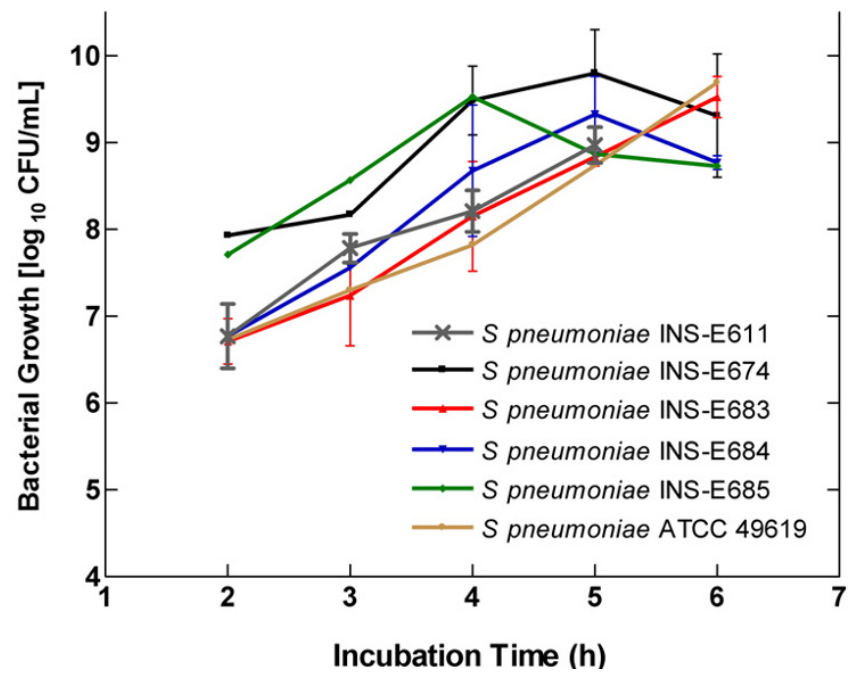

Figure 4

Growth curves of six strains of PRSP under optimized culture conditions. The high productivity of viable log-phased bacteria demonstrated for S. pneumoniae INSE6 II was reproduced with other 5 strains of PRSP, including diverse serotypes (Table I). More than $9.0 \log _{10} \mathrm{CFU} / \mathrm{mL}$ were obtained in a consistent manner with all strains (curves shown correspond to Tube 7, Phase 2). The variability between strains, evidenced by the time to reach maximal growth, is characteristic of $S$. pneumoniae.

further penicillin-resistant): INS-E611, E674 (blood isolates), E676, E678, E683, E684 (cerebrospinal fluid isolates); and two penicillin-susceptible strains: INS-E682 (blood isolate) and E685 (cerebrospinal fluid isolate). A standard strain of PRSP (S. pneumoniae ATCC 49619) was used as a control for all experiments (Table 1).

\section{Susceptibility testing}

MIC and MBC to penicillin, ceftriaxone and vancomycin were determined by broth microdilution following CLSI procedures [15]. MIC to the same antibiotics and to chloramphenicol, trimethoprim-sulfamethoxazole and erythromycin had been determined at the Colombian INS using identical methodology.

\section{Evaluation of baseline culture variables}

Strain INS-E611, a penicillin-resistant strain $(\mathrm{MIC}=2.0$ $\mu \mathrm{g} / \mathrm{mL}$ ), was selected to test a wide group of variables involved in bacterial growth that could have importance for $S$. pneumoniae. Once the growth dynamics under each variable were determined for this strain, the most productive conditions were standardized and applied to the remaining strains. Bacterial stocks were stored at $-70^{\circ} \mathrm{C}$ in two sets of 100 aliquots per strain. Cryoprotection media included $17 \%$ glycerol in trypticase soy broth for one set 
and skim milk for the other. The process from thawing to the end of variable evaluation was separated in three successive steps that we called phases: Phase 0 for resuscitating the frozen organism on to solid medium (two successive agar plates), Phase 1 for passing it to $10 \mathrm{ml}$ liquid medium a first time (five $16 \times 125$ glass tubes labeled 1 to 5 with successive 1:10 dilutions), and Phase 2 for a second transfer of log-phased cells into $10 \mathrm{ml}$ fresh liquid medium (three $16 \times 125$ glass tubes labeled 6 to 8 with successive 1:10 dilutions). The results were determined for all culture variables intervening at each phase and for the whole process by $\mathrm{OD}_{580 \mathrm{~nm}}$ (standard broths) and $\mathrm{OD}_{600 \mathrm{~nm}}$ (horse-blood supplemented broths) at 20-60 minutes intervals (SPECTRO 22, Labomed, Culver City, CA, USA); quantification of viable CFU per $\mathrm{mL}$ was made by dilution plating at least every hour during Phase 2.

These variables were tested by separate and combined at each step: cryoprotection media, addition of $0.5 \%$ yeast extract, and incubation time during Phase 0; inoculum size (number of CFU per tube), type of standard culture media, addition of $2 \%$ yeast extract and 2.5\% horse blood, initial pH adjustment to 7.8 (744 pH Meter, Metrohm Ltd., Switzerland) with $1 \mathrm{~N} \mathrm{NaOH}$ (EK CHEM, Germany), incubation time, and number of the most diluted tube with visible turbidity (used to inoculate Tube 6) during Phase 1; and type of standard culture media, addition of $2 \%$ yeast extract and $2.5 \%$ horse blood, $\mathrm{pH}$ adjustment to 7.8 every hour, incubation time, and bacterial growth rate from Tubes 6,7 and 8 during Phase 2. All three phases included incubation under $5 \% \mathrm{CO}_{2}$ atmosphere. Three types of standard culture media were evaluated in Phases 1 and 2: Brain Heart Infusion (BHI-Ox, Oxoid LTD, Basingstoke, Hampshire, UK), BBL ${ }^{\mathrm{TM}}$ Brain Heart Infusion (BBL-BHI, Becton Dickinson \& Co., Sparks, MD, USA) and Bacto ${ }^{\mathrm{TM}}$ Todd Hewitt Broth (THB, Becton Dickinson \& Co., Sparks, MD, USA). Horse blood was obtained directly by the research group and defibrinated, lysed, centrifuged and filtered following standard procedures [16]. Sterile quality control agar or broth was set simultaneously with the inoculated media in all culture processes, and incubation temperature was set to 37 and $35^{\circ} \mathrm{C}$ for experiments with standard and supplemented media, respectively. Experiments were repeated 2-3 times.

\section{Supplementation and optimization of culture conditions for PRSP}

The evaluation of baseline cultures variables allowed the creation of a culture protocol that was tested with all strains using the same phases. The results for the whole process for each strain were standardized by hourly determination of $\mathrm{OD}_{600 \mathrm{~nm}}$ and viable $\mathrm{CFU} / \mathrm{mL}$ count during Phase 2.

\section{Statistical analysis}

Data are presented as means and standard deviations. The significance of differences in productivity of various culture media along time was determined by Repeated Measures ANOVA. For differences in maximal production between these media, one way ANOVA was applied followed by the Bonferroni $t$ test. Data were stored, analyzed and graphed with Microsoft Excel v10.2 for Windows (Microsoft Corp., Seattle, WA, USA) and GraphPad Prism v4.0 for Windows (GraphPad Software, San Diego, CA, USA).

\section{Authors' contributions}

AVR carried out the microbiologic experiments, performed the analysis and interpretation of data and drafted the first versions of the manuscript. BES and MA carried out the microbiologic experiments and performed the analysis and interpretation of data. CAR carried out the microbiologic experiments, performed the analysis and interpretation of data and contributed in the study design. AFZ carried out the microbiologic experiments, performed the analysis and interpretation of data and drafted the first versions of the manuscript. OV conceived of the study, directed its design and coordination, obtained funding, directed data analysis, and re-wrote the final version of the manuscript. All authors contributed to the critical revision of the manuscript for important intellectual content and read and approved the final manuscript.

\section{Role of the Sponsor}

The funding organization had no role in the design and conduct of the study; collection, management, analysis, and interpretation of the data; and preparation, review, or approval of the manuscript.

\section{Acknowledgements}

We are grateful to Dr. Elizabeth Castañeda from Instituto Nacional de Salud, Bogotá, Colombia, for her kind assistance during the execution phase of the study and for providing the bacterial strains. This study was funded by research grant Colciencias III5-04-1298I and by University of Antioquia.

\section{References}

I. Austrian R: The pneumococcus at the millennium: not down, not out. J Infect Dis 1999, I 79(SuppI 2):S338-S34I.

2. Gonçalves VM, Zangirolami TC, Giordano RLC, Raw I, Tanizak MM, Giordano RC: Optimization of medium and cultivation conditions for capsular polysaccharide production by Streptococcus pneumoniae serotype 23F. Appl Microbiol Biotechnol 2002, 59:7|3-7|7.

3. Balachandran P, Hollingshead SK, Paton JC, Briles DE: The autolytic enzyme LytA of Streptococcus pneumoniae is not responsible for releasing pneumolysin. J Bacteriol 200I, I 83:3 I08-3 I I6.

4. Orihuela CJ, Gao G, McGee M, Yu J, Francis KP, Tuomanen E: Organ-specific models of Streptococcus pneumoniae disease. Scand J Infect Dis 2003, 35:647-652.

5. Rieux V, Carbon C, Azoulay-Dupuis E: Complex relationship between acquisition of beta-lactam resistance and loss of virulence in Streptococcus pneumoniae. J Infect Dis 200 I, I 84:66-72.

6. Baltz RH, Norris FH, Matsushima P, Dehoff BS, Rockey P, Porter G, Burgett S, Peery R, Hoskins J, Braverman L, Jenkins I, Solenberg P, 
Young M, McHenney MA, Rosteck PR, Skatrud PL: DNA sequence sampling and gene disruption for identification of new antibacterial targets in Streptococcus pneumoniae. In Streptococcus pneumoniae: molecular biology and mechanisms of disease Edited by: Tomasz A. New York: Mary Ann Liebert; 2000:33-44.

7. Rouff K, Whiley RA, Beighton D: Streptococcus. In Manual of clinical microbiology 7th edition. Edited by: Murray P, Baron E, Pfaller M, Tenover F, Yolken R. Washington, DC: ASM Press; 1999:283-296.

8. Musher D: Streptococcus pneumoniae. In Principles and practice of Infectious Diseases Volume 2. Fifth edition. Edited by: Mandell G, Bennett J, Dolin R. Philadelphia: Churchill Livingstone; 2000:2I29-2। 45.

9. Rotta J: Pyogenic haemolytic streptococci. In Bergey's Manual of Systematic Bacteriology Volume 2. Edited by: Sneath P, Mair N, Sharpe ME, Holt J. Baltimore: Williams \& Wilkins; 1986: 1052.

10. Lopez R, Garcia E, Garcia P, Garcia JL: Cell wall hydrolases. In The pneumococcus Edited by: Toumanen E, Mitchell T, Morrison D, Spratt B. Washington, DC: ASM Press; 2004:75-88.

II. Morrison D: Streptococcal competence for genetic transformation: regulation by peptide pheromones. In Streptococcus pneumoniae: molecular biology \& mechanisms of disease Edited by: Tomasz A. New York: Mary Ann Liebert; 2000:45-53.

12. Mazzola GJ, Mortensen JE, Millar LA, Poupard JA: The growth and survivability of Streptococcus pneumoniae clinical isolates subjected to various environmental conditions. Diag Microb Infect Dis 2003, 45: 153-164.

13. Canvin JR, Marvin AP, Sivakumaran M, Paton JC, Boulnois G], Andrew PW, Mitchell PJ: The role of pneumolysin and autolysin in the pathology of pneumonia and septicemia in mice infected with a type 2 pneumococcus. J Infect Dis 1995, I72: I 19-123.

14. Lopez R, Garcia E, Garcia P, Garcia JL: The pneumococcal cell wall degrading enzymes: a modular design to create new lysins? In Streptococcus pneumoniae: molecular biology \& mechanisms of disease Edited by: Tomasz A. New York: Mary Ann Liebert; 2000:197-209.

15. National Committee for Clinical Laboratory Standards: Performance standards for antimicrobial susceptibility testing; fourteenth informational supplement. M I00-SI 4 Volume 24. Issue I Wayne, PA; 2004.

16. Hindler JF, Hoechstein L, Howell A: Preparation of routine media and reagents used in antimicrobial susceptibility testing. In Clinical microbiology and procedures handbook Volume 2. 2nd edition. Edited by: Isenberg HD. Washington, DC: ASM Press; 2004. 5.I4.3.9

\section{Publish with Bio Med Central and every scientist can read your work free of charge}

"BioMed Central will be the most significant development for disseminating the results of biomedical research in our lifetime. "

Sir Paul Nurse, Cancer Research UK

Your research papers will be:

- available free of charge to the entire biomedical community

- peer reviewed and published immediately upon acceptance

- cited in PubMed and archived on PubMed Central

- yours - you keep the copyright

Submit your manuscript here:

http://www.biomedcentral.com/info/publishing_adv.asp
BiolMedcentral 